\title{
Finite-Time Stability of Solutions for Nonlinear $q$-Fractional Difference Coupled Delay Systems
}

\author{
Jingfeng Wang and Chuanzhi Bai iD \\ Department of Mathematics, Huaiyin Normal University, Huaian, Jiangsu 223300, China \\ Correspondence should be addressed to Chuanzhi Bai; czbai@hytc.edu.cn
}

Received 4 May 2021; Revised 22 September 2021; Accepted 29 September 2021; Published 10 November 2021

Academic Editor: Rigoberto Medina

Copyright (C) 2021 Jingfeng Wang and Chuanzhi Bai. This is an open access article distributed under the Creative Commons Attribution License, which permits unrestricted use, distribution, and reproduction in any medium, provided the original work is properly cited.

In this paper, we investigate and prove a new discrete $q$-fractional version of the coupled Gronwall inequality. By applying this result, the finite-time stability criteria of solutions for a class of nonlinear $q$-fractional difference coupled delay systems are obtained. As an application, an example is provided to demonstrate the effectiveness of our result.

\section{Introduction}

The $q$-difference equations have numerous applications in diverse fields in recent years and have gained intensive interest [1-4]. For more details on $q$-calculus, we recommend the readers to [5]. In the last two decades, the fractional difference equations have recently received considerable attention in many fields of science and engineering, see [6-9] and the references therein. We know that the $q$-fractional difference equations can be used as a bridge between fractional difference equations and $q$-difference equations, and there are many papers on this research direction which have been appeared in [10-16]. And, we recommend [17] and the papers cited therein.

For $0<q<1$, we define the time scale $\mathbb{T}_{q}=$ $\left\{q^{n}: n \in \mathbb{Z}\right\} \cup\{0\}$, where $\mathbb{Z}$ is the set of integers. For $a=q^{n_{0}}$ and $n_{0} \in \mathbb{Z}$, we denote $\mathbb{T}_{a}=[a, \infty)_{q}=\left\{q^{-i} a: i=0,1,2, \ldots\right\}$.

In [18], Abdeljawad and Alzabut established a discrete $q$-fractional version of the Gronwall-type inequality as follows:

Theorem 1 (see [18]). Let $\alpha>0, u$ and $\mu$ be nonnegative real valued functions such that $0 \leq \mu(t)<1 / t^{\alpha}(1-q)^{\alpha}$, for all $t \in \mathbb{T}_{a}$, and

$$
u(t) \leq u(a)+{ }_{q} \nabla_{a}^{-\alpha} u(t) \mu(t)
$$

Then,

$$
u(t) \leq u(a) \sum_{k=0}^{\infty}{ }_{q} E_{\mu}^{k} 1,
$$

where ${ }_{q} E_{\mu}^{k} 1=\mu^{k}(t-a)_{q}^{k \alpha} / \Gamma_{q}(k \alpha+1)$.

Abdeljawad et al., in [19], extended the above inequality and obtained the following generalized $q$-fractional Gronwall-type inequality.

Theorem 2 (see [19]). Let $\alpha>0, u$ and $v$ be nonnegative functions, and $w(t)$ be nonnegative and nondecreasing function, for $t \in[a, \infty)_{q}$, such that $w(t) \leq M$, where $M$ is a constant. If

$$
u(t) \leq v(t)+w(t)_{q} \nabla_{a}^{-\alpha} u(t)
$$

then

$$
u(t) \leq v(t)+\sum_{k=1}^{\infty}\left(w(t) \Gamma_{q}(\alpha)\right)_{q}^{k} \nabla_{a}^{-k \alpha} \nu(t) .
$$

Based on the above result, Abdeljawad et al. investigated the following nonlinear delay $q$-fractional difference system: 


$$
\begin{cases}{ }_{q} C_{a}^{\alpha} x(t)=A_{0} x(t)+A_{1} x(\tau t)+f(t, x(t), x(\tau t)), & t \in[a, \infty){ }_{q}, \\ x(t)=\phi(t), & t \in \mathbb{\mathbb { V }}_{\tau},\end{cases}
$$

where ${ }_{q} C_{a}^{\alpha}$ means the Caputo fractional difference of order $\alpha$, $\mathbb{\square}_{\tau}=\left\{\tau a, q^{-1} \tau a, q^{-2} \tau a, \ldots, a\right\}, \quad \tau=q^{d} \in \mathbb{T}_{q}$, with $d \in \mathbb{N}_{0}=$ $\{0,1,2, \ldots\}$.

An interesting topic in control theory is finite-time control, and the objective of finite-time control is to design a control law, making the system state converge to the origin in finite time. In [20], Sun et al. developed the finite-time state feedback stabilisation scheme for nonlinear time-delay systems with high-order and low-order nonlinearities. Recently, Wang and Xiang [21, 22] presented a finite-time output feedback control scheme for a class of nonlinear systems and nonlinear time-delay in the $p$-normal form, respectively. In [23], Modiri and Mobayen studied the synchronization of fractional-order uncertain chaotic systems in the finite time. Mofid et al., in [24], considered the sliding mode disturbance observer control of a class of fractional-order chaotic systems by using adaptive synchronization. Moreover, the observer-based state feedback stabilizer design for a class of chaotic systems and the fixedtime attitude control for a flexible spacecraft in the presence of actuator faults, external disturbances, and coupling effect of flexible modes have been considered in $[25,26]$, respectively.

On the contrary, finite-time stability is a method which is much valuable to analyze the transient behavior of nature of a system within a finite interval of time. In recent decades, the finite-time stability analysis of fractional differential systems have recently considerable attention, see, for instance, [27-31] and the references therein. However, till now, few researchers focus on finite-time stability of fractional delay difference systems.

Motivated by the above works, we will to extend the $q$-fractional Gronwall-type inequality (Theorem 2) to coupled $q$-fractional Gronwall inequality. As an application, we establish a finite-time stability criterion of the following nonlinear coupled delay $q$-fractional difference system:

$$
\begin{cases}{ }_{q} C_{a}^{\alpha} x(t)=A_{0} y(t)+A_{1} y(\tau t)+f(t, y(t), y(\tau t)), & t \in[a, 1]_{q}, \\ { }_{q} C_{a}^{\alpha} y(t)=B_{0} x(t)+B_{1} x(\tau t)+g(t, x(t), x(\tau t)), & t \in[a, 1]_{q}, \\ x(t)=\phi(t), \quad y(t)=\psi(t), & t \in \mathbb{\mathbb { V }}_{\tau},\end{cases}
$$

where $[a, 1]_{q}=[a, 1] \cap \mathbb{T}_{a}, \mathbb{q}_{\tau}=\left\{\tau a, q^{-1} \tau a, q^{-2} \tau a, \ldots, a\right\}$, $\tau=q^{d} \in \mathbb{T}_{q}$ with $d \in \mathbb{N}_{0}=\{0,1,2, \ldots\},{ }_{q} C_{a}^{\alpha}$ and ${ }_{q} C_{a}^{\beta}$ mean the Caputo fractional difference of order $\alpha \in(0,1)$ and order $\beta \in(0,1)$, respectively, and the constant matrices $A_{0}, A_{1}, B_{0}$, and $B_{1}$ are of appropriate dimensions.

In this paper, the coupled $q$-fractional Gronwall inequality is studied and given for the first time, which is a powerful tool and method to deal with finite-time stability and other stability of nonlinear coupled delay $q$-fractional difference systems. And, we studied the finite-time stability of a class of nonlinear coupled delay $q$-fractional difference system by using this inequality.
The organization of this paper is given as follows. In Section 2, we give some notations, definitions, and preliminaries. Section 3 is devoted to proving a coupled $q$-fractional Gronwall inequality. In Section 4, the finite-time stability theorem of nonlinear coupled delay $q$-fractional difference system is proved. In Section 5, an example is given to illustrate our theoretical result. Finally, the paper is concluded in Section 6.

\section{Preliminaries}

In this section, we provided some basic definitions and lemmas which are used in the sequel. $f$ by

Let $f: \mathbb{T}_{q} \longrightarrow \mathbb{R}$, and we define the nabla $q$-derivative of

$$
\nabla_{q} f(t)=\frac{f(t)-f(q t)}{(1-q) t}, \quad t \in \mathbb{T}_{q} \backslash\{0\} .
$$

The nabla $q$-integral of $f$ has the following form:

$$
\int_{0}^{t} f(s) \nabla_{q} s=(1-q) t \sum_{i=0}^{\infty} q^{i} f\left(t q^{i}\right)
$$

and for $0 \leq a \in \mathbb{T}_{q}$,

$$
\int_{a}^{t} f(s) \nabla_{q} s=\int_{0}^{t} f(s) \nabla_{q} s-\int_{0}^{a} f(s) \nabla_{q} s .
$$

The definition of the $q$-factorial function for a nonpositive integer $\alpha$ is given by

$$
(t-s)_{q}^{\alpha}=t^{\alpha} \prod_{i=0}^{\infty} \frac{1-(s / t) q^{i}}{1-(s / t) q^{i+\alpha}}
$$

For a function $f: \mathbb{T}_{q} \longrightarrow \mathbb{R}$, the left $q$-fractional integral ${ }_{q} \nabla_{a}^{-\alpha}$ of order $\alpha \neq 0,-1,-2, \ldots$ and starting at $0<a \in \mathbb{T}_{q}$ is defined by

$$
\nabla_{a}^{-\alpha} f(t)=\frac{1}{\Gamma_{q}(\alpha)} \int_{a}^{t}(t-\mathrm{qs})_{q}^{\alpha-1} f(s) \nabla_{q} s,
$$

where

$$
\Gamma_{q}(\alpha+1)=\frac{1-q^{\alpha}}{1-q} \Gamma_{q}(\alpha), \quad \Gamma_{q}(1)=1, \alpha>0 .
$$

Definition 1 (see [11]). Let $0<\alpha \notin \mathbb{N}$. Then, the Caputo left $q$-fractional derivative of order $\alpha$ of a function $f$ defined on $\mathbb{T}_{q}$ is defined by

$$
\begin{aligned}
{ }_{q} C_{a}^{\alpha} f(t) & :={ }_{q} \nabla_{a}^{-(n-\alpha)} \nabla_{q}^{n} f(t) \\
& =\frac{1}{\Gamma_{q}(n-\alpha)} \int_{a}^{t}(t-\mathrm{qs})_{q}^{n-\alpha-1} \nabla_{q}^{n} f(s) \nabla_{q} s
\end{aligned}
$$

where $n=[\alpha]+1$.

Lemma 1 (see [11]). Let $\alpha>0$ and $f$ be defined in a suitable domain. Thus, 


$$
{ }_{q} \nabla_{a}^{-\alpha}{ }_{q} C_{a}^{\alpha} f(t)=f(t)-\sum_{k=0}^{n-1} \frac{(t-a)_{q}^{k}}{\Gamma_{q}(k+1)} \nabla_{q}^{k} f(a),
$$

and if $0<\alpha \leq 1$, we have

$$
{ }_{q} \nabla_{a}^{-\alpha} C_{a}^{\alpha} f(t)=f(t)-f(a) .
$$

The following identity plays a crucial role in solving the linear $q$-fractional equations:

$$
q_{a} \nabla_{a}^{-\alpha}(x-a)_{q}^{\mu}=\frac{\Gamma_{q}(\mu+1)}{\Gamma_{q}(\alpha+\mu+1)}(x-a)_{q}^{\mu+\alpha} \quad 0<a<x<b,
$$

where $\alpha \in \mathbb{R}^{+}$and $\mu \in(-1, \infty)$. The $q$-analog of the Mittag-Leffler function with double index $(\alpha, \beta)$ is introduced as follows.

Definition 2 (see [11]). For $z, z_{0} \in \mathbb{C}$ and $\mathfrak{R}(\alpha)>0$, the $q$-Mittag-Leffler function is defined by

$$
{ }_{q} E_{\alpha, \beta}\left(\lambda, z-z_{0}\right)=\sum_{k=0}^{\infty} \lambda^{k} \frac{\left(z-z_{0}\right)_{q}^{\alpha k}}{\Gamma_{q}(\alpha k+\beta)} .
$$

In the case $\beta=1$, we utilize ${ }_{q} E_{\alpha}\left(\lambda, z-z_{0}\right)={ }_{q} E_{\alpha, 1}$ $\left(\lambda, z-z_{0}\right)$.
Moreover, the modified $q$-Mittag-Leffler function is used in [19] as follows:

$$
q_{\alpha, \beta}^{e}\left(\lambda, z-z_{0}\right)=\sum_{k=0}^{\infty} \lambda^{k} \frac{\left(z-z_{0}\right)_{q}^{\alpha k+(\beta-1)}}{\Gamma_{q}(\alpha k+\beta)} .
$$

\section{A Generalized Coupled $q$-Fractional Gronwall Inequality}

In this section, we give and prove the following a generalized coupled $q$-fractional Gronwall inequality, which extend a generalized $q$-fractional Gronwall inequality in Theorem 2 .

Theorem 3. Assume that $u(t), v(t)$, and $g_{i}(t)(i=1,2)$ are nonnegative functions for $t \in \mathbb{T}_{a}$. Let $w_{i}(t)(i=1,2)$ be nonnegative and nondecreasing functions for $t \in \mathbb{T}_{a}$ with $w_{i}(t) \leq M_{i}$, where $M_{i}$ are constants $(i=1,2)$. If

$$
\begin{cases}u(t) \leq g_{1}(t)+w_{1}(t)_{q} \nabla_{a}^{-\alpha} v(t), & t \in[a, 1]_{q}, \\ v(t) \leq g_{2}(t)+w_{2}(t)_{q} \nabla_{a}^{-\beta} u(t), & t \in[a, 1]_{q},\end{cases}
$$

and

$$
M_{1} M_{2}(1-q)^{\alpha+\beta}<1,
$$

hold, then

$$
\begin{aligned}
u(t) \leq & g_{1}(t)+\frac{w_{1}(t)}{\Gamma_{q}(\alpha)} \int_{a}^{t}(t-\mathrm{q} s)_{q}^{\alpha-1} g_{2}(s) \nabla_{q} s \\
& +\sum_{k=1}^{\infty} w_{1}(t)^{k} w_{2}(t)^{k} \nabla_{a}^{-k(\alpha+\beta)}\left(g_{1}(t)+\frac{w_{1}(t)}{\Gamma_{q}(\alpha)} \int_{q}^{t}(t-\mathrm{q} s)_{q}^{\alpha-1} g_{2}(s) \nabla_{q} s\right),
\end{aligned}
$$

and

$$
\begin{aligned}
v(t) \leq & g_{2}(t)+\frac{w_{2}(t)}{\Gamma_{q}(\beta)} \int_{a}^{t}(t-\mathrm{qs})_{q}^{\beta-1} g_{1}(s) \nabla_{q} s \\
& +\sum_{k=1}^{\infty} w_{1}(t)^{k} w_{2}(t)^{k}{ }_{q} \nabla_{a}^{-k(\alpha+\beta)}\left(g_{2}(t)+\frac{w_{2}(t)}{\Gamma_{q}(\beta)} \int_{a}^{t}(t-\mathrm{qs})_{q}^{\beta-1} g_{1}(s) \nabla_{q} s\right) .
\end{aligned}
$$

Proof. Let

$$
\operatorname{Av}(t)=\frac{w_{1}(t)}{\Gamma_{q}(\alpha)} \int_{a}^{t}(t-\mathrm{qs})_{q}^{\alpha-1} v(s) \nabla_{q} s, \quad t \in \mathbb{T}_{a},
$$

and

$$
\mathrm{Bu}(t)=\frac{w_{2}(t)}{\Gamma_{q}(\beta)} \int_{a}^{t}(t-\mathrm{qs})_{q}^{\beta-1} u(s) \nabla_{q} s, \quad t \in \mathbb{T}_{a} .
$$

According to (19), one has

$$
u(t) \leq g_{1}(t)+\operatorname{Av}(t), v(t) \leq g_{2}(t)+\mathrm{Bu}(t) .
$$


By (25) and the monotonicity of the operators $A$ and $B$, we obtain

$$
\begin{aligned}
u(t) & \leq g_{1}(t)+A\left(g_{2}(t)+\mathrm{Bu}(t)\right)=g_{1}(t)+\mathrm{Ag}_{2}(t)+\mathrm{ABu}(t) \\
& \leq g_{1}(t)+A g_{2}(t)+\mathrm{AB}\left[g_{1}(t)+\operatorname{Ag}_{2}(t)+\mathrm{ABu}(t)\right] \\
& =g_{1}(t)+\operatorname{ABg}_{1}(t)+\operatorname{Ag}_{2}(t)+\operatorname{ABAg}_{2}(t)+(\mathrm{AB})^{2} u(t) \\
& \leq \sum_{k=0}^{n-1}(\mathrm{AB})^{k} g_{1}(t)+\sum_{k=0}^{n-1}(\mathrm{AB})^{k} \operatorname{Ag}_{2}(t)+(\mathrm{A})^{n} u(t), \quad t \in \mathbb{T}_{a} .
\end{aligned}
$$

Similarly, we obtain

$v(t) \leq \sum_{k=0}^{n-1}(\mathrm{BA})^{k} g_{2}(t)+\sum_{k=0}^{n-1}(\mathrm{BA})^{k} \mathrm{Bg}_{1}(t)+(\mathrm{BA})^{n} v(t), \quad t \in \mathbb{T}_{a}$, where $(\mathrm{AB})^{0} g_{1}(t)=g_{1}(t)$ and $(\mathrm{BA})^{0} g_{2}(t)=g_{2}(t)$.

In the following, we will prove that

$$
\begin{aligned}
& (\mathrm{AB})^{n} u(t) \leq w_{1}(t)^{n} w_{2}(t)_{q}^{n} \nabla_{a}^{-n(\alpha+\beta)} u(t), \\
& (\mathrm{BA})^{n} v(t) \leq w_{1}(t)^{n} w_{2}(t)^{n}{ }_{q} \nabla_{a}^{-n(\alpha+\beta)} v(t),
\end{aligned}
$$

where $t \in \mathbb{T}_{a}$ and

$$
\lim _{n \longrightarrow \infty}(\mathrm{AB})^{n} u(t)=0, \lim _{n \longrightarrow \infty}(\mathrm{BA})^{n} v(t)=0 .
$$

We know that (28) and (29) are true for $n=1$. In fact, one has

$$
\begin{aligned}
\operatorname{ABu}(t) & =A(\mathrm{Bu}(t))=\frac{w_{1}(t)}{\Gamma_{q}(\alpha)} \int_{a}^{t}(t-\mathrm{qs})_{q}^{\alpha-1} \mathrm{Bu}(s) \nabla_{q} s \\
& =\frac{w_{1}(t)}{\Gamma_{q}(\alpha) \Gamma_{q}(\beta)} \int_{a}^{t}(t-\mathrm{qs})_{q}^{\alpha-1} w_{2}(s) \int_{a}^{s}(t-\mathrm{qr})_{q}^{\beta-1} u(r) \nabla_{q} r \nabla_{q} s \\
& \leq \frac{w_{1}(t) w_{2}(t)}{\Gamma_{q}(\alpha) \Gamma_{q}(\beta)} \int_{a}^{t}(t-\mathrm{qs})_{q}^{\alpha-1} \int_{a}^{s}(t-\mathrm{qr})_{q}^{\beta-1} u(r) \nabla_{q} r \nabla_{q} s \\
& =\frac{w_{1}(t) w_{2}(t)}{\Gamma_{q}(\alpha) \Gamma_{q}(\beta)} \int_{a}^{t} \int_{r}^{t}(t-\mathrm{qs})_{q}^{\alpha-1}(s-\mathrm{qr})_{q}^{\beta-1} u(r) \nabla_{q} r \nabla_{q} s \\
& =\frac{w_{1}(t) w_{2}(t)}{\Gamma_{q}(\beta)} \int_{a}^{t}\left[\frac{1}{\Gamma_{q}(\alpha)} \int_{r}^{t}(t-\mathrm{qs})_{q}^{\alpha-1}(s-\mathrm{qr})_{q}^{\beta-1} \nabla_{q} s\right] u(r) \nabla_{q} r \\
& =\frac{w_{1}(t) w_{2}(t)}{\Gamma_{q}(\beta)} \int_{a}^{t} \nabla_{q r}^{-\alpha}(t-\mathrm{qr})_{q}^{\beta-1} u(r) \nabla_{q}^{r},
\end{aligned}
$$

where ${ }_{q} \nabla_{q r}^{-\alpha} u(t)=1 / \Gamma_{q}(\alpha) \int_{q r}^{t}(t-\mathrm{qs})_{q}^{\alpha-1} u(s) \nabla_{q} s$ has been used. By (16), we have

$$
\begin{aligned}
q_{a} \nabla_{a}^{-\alpha}(x-a)_{q}^{\mu}= & \frac{\Gamma_{q}(\mu+1)}{\Gamma_{q}(\alpha+\mu+1)}(x-a)_{q}^{\mu+\alpha} \\
& (0<a<x<b), \mu>-1 .
\end{aligned}
$$$$
\operatorname{ABu}(t) \leq \frac{w_{1}(t) w_{2}(t)}{\Gamma_{q}(\beta)} \int_{a}^{t} \frac{\Gamma_{q}(\beta)}{\Gamma_{q}(\alpha+\beta)}(t-q r)_{q}^{\alpha+\beta-1} u(r) \nabla_{q} r
$$$$
=\frac{w_{1}(t) w_{2}(t)}{\Gamma_{q}(\alpha+\beta)} \int_{a}^{t}(t-\mathrm{qr})_{q}^{\alpha+\beta-1} u(r) \nabla_{q} r
$$$$
=w_{1}(t) w_{2}(t)_{q} \nabla_{a}^{-(\alpha+\beta)} u(t) .
$$

\footnotetext{
Combining (31) with (32), one has
}

Similarly, one has 


$$
\operatorname{BAv}(t) \leq w_{1}(t) w_{2}(t)_{q} \nabla_{a}^{-(\alpha+\beta)} v(t) .
$$

Thus, (28) and (29) are valid for $n=1$. Assume that (28) and (29) are true, for $n=k$, which are

$$
\begin{aligned}
(\mathrm{AB})^{k+1} u(t) & =\mathrm{AB}\left((\mathrm{AB})^{k} u(t)\right) \\
& \leq \frac{w_{1}(t) w_{2}(t)}{\Gamma_{q}(\alpha+\beta)} \int_{a}^{t}(t-\mathrm{qs})_{q}^{\alpha+\beta-1} \int_{a}^{s} \frac{w_{1}(s)^{k} w_{2}(s)^{k}}{\Gamma_{q}(k(\alpha+\beta))}(t-\mathrm{qr})_{q}^{k(\alpha+\beta)-1} u(r) \nabla_{q} r \nabla_{q} s \\
& \leq \frac{w_{1}(t)^{k+1} w_{2}(t)^{k+1}}{\Gamma_{q}(k(\alpha+\beta))} \int_{a}^{t}\left[\int_{r}^{t} \frac{1}{\Gamma_{q}(\alpha+\beta)}(t-\mathrm{qs})_{q}^{\alpha+\beta-1}(s-\mathrm{qr})_{q}^{k(\alpha+\beta)-1} \nabla_{q} s\right] u(r) \nabla_{q} r \\
& =\frac{w_{1}(t)^{k+1} w_{2}(t)^{k+1}}{\Gamma_{q}(k(\alpha+\beta))} \int_{a}^{t} \nabla_{q r}^{-(\alpha+\beta)}(t-\mathrm{qr})_{q}^{k(\alpha+\beta)-1} u(r) \nabla_{q}^{r},
\end{aligned}
$$

been used. By using (16) and (37), we obtain

$$
\begin{aligned}
(\mathrm{AB})^{k+1} u(t) & \leq \frac{w_{1}(t)^{k+1} w_{2}(t)^{k+1}}{\Gamma_{q}(k(\alpha+\beta))} \int_{a}^{t}(t-\mathrm{qr})_{q}^{(k+1)(\alpha+\beta)-1} \frac{\Gamma_{q}(k(\alpha+\beta))}{\Gamma_{q}(k+1)(\alpha+\beta)} u(r) \nabla_{q} r \\
& =\frac{w_{1}(t)^{k+1} w_{2}(t)^{k+1}}{\Gamma_{q}((k+1)(\alpha+\beta))} \int_{a}^{t}(t-\mathrm{qr})_{q}^{(k+1)(\alpha+\beta)-1} u(r) \nabla_{q} r \\
& =w_{1}(t)^{k+1} w_{2}(t)^{k+1} \nabla_{a}^{-(k+1)(\alpha+\beta)} u(t) .
\end{aligned}
$$

Similarly, we can obtain

$$
(\mathrm{BA})^{k+1} v(t) \leq w_{1}(t)^{k+1} w_{2}(t)^{k+1} \nabla_{a}^{-(k+1)(\alpha+\beta)} v(t) .
$$

Thus, (28) and (29) are proved.

Using Stirling's formula of the q-gamma function [32] yields that

$$
\Gamma_{q}(x)=[2]_{q}^{1 / 2} \Gamma_{q^{2}}\left(\frac{1}{2}\right)(1-q)^{(1 / 2)-x} e^{\theta q^{x} /(1-q)-q^{x}}, \quad 0<\theta<1,
$$

that is,

$$
\Gamma_{q}(x) \sim D(1-q)^{(1 / 2)-x}, \quad x \longrightarrow \infty,
$$

where $D=[2]_{q}^{1 / 2} \Gamma_{q^{2}}(1 / 2)$. Moreover, if $t>a>0$ and $\gamma>0(\gamma$ is not a positive integer), then $1-a / t q^{j}<1-a / t q^{\gamma+j}$, for each $j=0,1, \ldots$, and

$$
(t-a)_{q}^{\gamma}=t^{\gamma} \prod_{j=0}^{\infty} \frac{1-a / t q^{j}}{1-a / t q^{\gamma+j}}<t^{\gamma} .
$$

Applying the first mean value theorem for definite integrals [33], (41) and (42), and $w_{1}(t)<M_{1}$ and $w_{2}(t)<M_{2}$, there exists a $\xi \in[a, 1]_{q}$ such that 


$$
\begin{aligned}
\lim _{n \longrightarrow \infty}(\mathrm{AB})^{n} u(t) & \leq \lim _{n \longrightarrow \infty} u(\xi) \frac{M_{1}^{n} M_{2}^{n}}{\Gamma_{q}(n(\alpha+\beta))} \int_{a}^{t}(t-\mathrm{qr})_{q}^{n(\alpha+\beta)-1} \nabla_{q} r \\
& =\lim _{n \longrightarrow \infty} u(\xi) \frac{M_{1}^{n} M_{2}^{n}}{\Gamma_{q}(n(\alpha+\beta)+1)}(t-a)_{q}^{n(\alpha+\beta)} \\
& \leq \lim _{n \longrightarrow \infty} u(\xi) \frac{M_{1}^{n} M_{2}^{n}}{\Gamma_{q}(n(\alpha+\beta)+1)} t^{n(\alpha+\beta)} \\
& =\lim _{n \longrightarrow \infty} u(\xi) \frac{M_{1}^{n} M_{2}^{n}}{D(1-q)^{1 / 2-(n(\alpha+\beta)+1)}} t^{n(\alpha+\beta)} \\
& \leq \lim _{n \longrightarrow \infty} u(\xi) \frac{M_{1}^{n} M_{2}^{n}}{D(1-q)^{1 / 2-(n(\alpha+\beta)+1)}} \\
& =\lim _{n \longrightarrow \infty} \frac{u(\xi) \sqrt{1-q}}{D}\left[M_{1} M_{2}(1-q)^{\alpha+\beta}\right]^{n} .
\end{aligned}
$$

From (20), for each $t \in[a, 1]_{q}$, one has

$$
\left[M_{1} M_{2}(1-q)^{\alpha+\beta}\right]^{n} \longrightarrow 0, \quad \text { as } n \longrightarrow \infty
$$

Let $n \longrightarrow \infty$ in (26); with the help of the semigroup property ${ }_{q} \nabla_{a}^{-\alpha} \nabla_{a}^{-\mu}={ }_{q} \nabla_{a}^{-(\alpha+\mu)}$ and the definition of $A$ and $B$, one obtains

Thus, $(\mathrm{AB})^{n} u(t) \longrightarrow 0$ as $n \longrightarrow \infty$, Similarly, we can obtain that $(\mathrm{BA})^{n} u(t) \longrightarrow 0$ as $n \longrightarrow \infty$, for each $t \in \mathbb{T}_{a}$. Therefore, (30) is proved.

$$
\begin{aligned}
u(t) \leq & g_{1}(t)+A g_{2}(t)+\sum_{k=1}^{\infty}(\mathrm{AB})^{k} g_{1}(t)+\sum_{k=1}^{\infty}(\mathrm{AB})^{k} A g_{2}(t) \\
= & g_{1}(t)+\frac{w_{1}(t)}{\Gamma_{q}(\alpha)} \int_{a}^{t}(t-\mathrm{qs})_{q}^{\alpha-1} g_{2}(s) \nabla_{q} s \\
& +\sum_{k=1}^{\infty} w_{1}(t)^{k} w_{2}(t)^{k} \nabla_{a}^{-k(\alpha+\beta)}\left(g_{1}(t)+\frac{w_{1}(t)}{\Gamma_{q}(\alpha)} \int_{a}^{t}(t-\mathrm{qs})_{q}^{\alpha-1} g_{2}(s) \nabla_{q} s\right)
\end{aligned}
$$

Similarly, let $n \longrightarrow \infty$ in (27), and we obtain (22). This completes the proof.

Corollary 1. Under the hypothesis of Theorem 3, let $g_{1}(t)$ and $g_{2}(t)$ be two nondecreasing functions on $t \in \mathbb{T}_{a}$. Then,

$$
\begin{aligned}
u(t) \leq & g_{1}(t)_{q} E_{\alpha+\beta}\left(w_{1}(t) w_{2}(t), t-a\right) \\
& +w_{1}(t) g_{2}(t)_{q} e_{\alpha+\beta, \alpha+1}\left(w_{1}(t) w_{2}(t), t-a\right)
\end{aligned}
$$

and

$$
\begin{aligned}
v(t) \leq & g_{2}(t)_{q} E_{\alpha+\beta}\left(w_{1}(t) w_{2}(t), t-a\right) \\
& +w_{2}(t) g_{1}(t)_{q} e_{\alpha+\beta, \beta+1}\left(w_{1}(t) w_{2}(t), t-a\right) .
\end{aligned}
$$

Proof. By (16) and the assumption that $g_{1}(t), g_{2}(t)$, and $w_{1}(t)$ are three nondecreasing functions for $t \in \mathbb{T}_{a}$, we have

$$
q_{a}^{-\alpha} g_{2}(t) \leq g_{2}(t)_{q} \nabla_{a}^{-\alpha} 1=\frac{g_{2}(t)}{\Gamma_{q}(\alpha+1)}(t-a)_{q}^{\alpha},
$$

and

$$
\begin{aligned}
& { }_{q} \nabla_{a}^{-k(\alpha+\beta)}\left(g_{1}(t)+w_{1}(t)_{q} \nabla_{a}^{-\alpha} g_{2}(t)\right) \\
\leq & \left(g_{1}(t)+w_{1}(t)_{q} \nabla_{a}^{-\alpha} g_{2}(t)\right)_{q} \nabla_{a}^{-k(\alpha+\beta)} 1 \\
= & \left(g_{1}(t)+w_{1}(t)_{q} \nabla_{a}^{-\alpha} g_{2}(t)\right) \frac{(t-a)_{q}^{k(\alpha+\beta)}}{\Gamma_{q}(k(\alpha+\beta)+1)} .
\end{aligned}
$$


Thus, from (21), (48), and (49), one can conclude that

$$
\begin{aligned}
u(t) \leq & \left(g_{1}(t)+w_{1}(t) g_{2}(t)_{q} \nabla_{a}^{-\alpha} 1\right)\left[1+\sum_{k=1}^{\infty} w_{1}(t)^{k} w_{2}(t)_{q}^{k} \nabla_{a}^{-k(\alpha+\beta)} 1\right] \\
= & g_{1}(t)\left[1+\sum_{k=1}^{\infty} w_{1}(t)^{k} w_{2}(t)^{k}{ }_{q} \nabla_{a}^{-k(\alpha+\beta)} 1\right]+\frac{w_{1}(t) g_{2}(t)}{\Gamma_{q}(\alpha+1)}(t-a)_{q}^{\alpha} \\
& +w_{1}(t) g_{2}(t) \sum_{k=1}^{\infty} w_{1}(t)^{k} w_{2}(t)^{k}{ }_{q} \nabla_{a}^{-k(\alpha+\beta)}{ }_{q} \nabla_{a}^{-\alpha} 1 \\
= & g_{1}(t) \sum_{k=0}^{\infty} \frac{\left(w_{1}(t) w_{2}(t)\right)^{k}(t-a)_{q}^{k(\alpha+\beta)}}{\Gamma_{q}(k(\alpha+\beta)+1)}+\frac{w_{1}(t) g_{2}(t)}{\Gamma_{q}(\alpha+1)}(t-a)_{q}^{\alpha} \\
& +w_{1}(t) g_{2}(t) \sum_{k=1}^{\infty} w_{1}(t)^{k} w_{2}(t)^{k}{ }_{q} \nabla_{a}^{-k(\alpha+\beta)-\alpha} 1 \\
= & g_{1}(t)_{q} E_{\alpha+\beta}\left(w_{1}(t) w_{2}(t), t-a\right)+\frac{w_{1}(t) g_{2}(t)}{\Gamma_{q}(\alpha+1)}(t-a)_{q}^{\alpha} \\
= & g_{1}(t)_{q} E_{\alpha+\beta}\left(w_{1}(t) w_{2}(t), t-a\right)+w_{1}(t) g_{2}(t) \sum_{k=0}^{\infty} \frac{\left(w_{1}(t) w_{2}(t)\right)^{k}(t-a)_{q}^{k(\alpha+\beta)+\alpha}}{\Gamma_{q}(k(\alpha+\beta)+\alpha+1)} \\
& +w_{1}(t) g_{2}(t) \sum_{k=1}^{\infty} \frac{\left(w_{1}(t) w_{2}(t)\right)^{k}(t-a)_{q}^{k(\alpha+\beta)+\alpha}}{\Gamma_{q}(k(\alpha+\beta)+\alpha+1)}
\end{aligned}
$$

Similarly,we can obtain (47) holds.

\section{Main Result}

Throughout this paper, we make the following assumptions:
(H1) $f, g \in D\left(\mathbb{T}_{a} \times \mathbb{R}^{n}, \mathbb{R}^{n}\right)$ are two Lipschitz-type functions. That is, for any $x, y, u, v: \mathbb{T}_{\tau a} \longrightarrow \mathbb{R}^{n}$, there exist two positive constants $L_{1}, L_{2}>0$ such that

$$
\begin{aligned}
& \|f(t, y(t), y(\tau t))-g(t, v(t), t v n(\tau t))\| \leq L_{1}(\|y(t)-v(t)\|+\|y(\tau t)-v(\tau t)\|), \\
& \|g(t, x(t), x(\tau t))-g(t, u(t), \operatorname{tun}(\tau t))\| \leq L_{2}(\|x(t)-u(t)\|+\|x(\tau t)-u(\tau t)\|)
\end{aligned}
$$

For $t \in[a, 1]_{q}$,

$(\mathrm{H} 2) f(t, 0,0)=\underbrace{[0,0, \ldots, 0]}_{n}, \quad g(t, 0,0)=\underbrace{[0,0, \ldots, 0]}_{n}$,

(H3) $\left(\left\|A_{0}\right\|+\left\|A_{1}\right\|+2 L_{1}\right)\left(\left\|B_{0}\right\|+\left\|B_{1}\right\|+2 L_{2}\right)(1-q)^{\alpha+\beta}<1$.
Let us denote $\quad\|(\phi, \psi)\|=\max \left\{\max _{t \in \cup_{\tau}}\|\phi(t)\|\right.$, $\left.\max _{t \in \mathbb{V}_{\tau}}\|\psi(t)\|\right\}$.

Definition 3. System (6) is finite-time stable with respect to $\{\delta, \varepsilon, T\}$, with $\delta<\epsilon$, if and only if $\|(\phi, \psi)\|<\delta$ implies $\|(x(t), y(t))\|=\max \{\|x(t)\|,\|y(t)\|\}<\varepsilon, \forall t \in \mathbb{T}_{\tau a}$. 
Theorem 4. Assume that conditions (H1)-(H3) hold. Then, system (6) is finite-time stable if the following conditions are satisfied:

$$
\begin{aligned}
& \left(1+\frac{\left\|A_{1}\right\|+L_{1}}{\Gamma_{q}(\alpha+1)}(t-a)_{q}^{\alpha}\right)_{q} E_{\alpha+\beta}(c, t-a) \\
& +\left(\left\|A_{0}\right\|+\left\|A_{1}\right\|+2 L_{1}\right)\left(1+\frac{\left\|B_{1}\right\|+L_{2}}{\Gamma_{q}(\beta+1)}(t-a)_{q}^{\beta}\right)_{q} e_{\alpha+\beta, \alpha+1}(c, t-a)<\frac{\varepsilon}{\delta},
\end{aligned}
$$

and

$$
\begin{aligned}
& \left(1+\frac{\left\|B_{1}\right\|+L_{2}}{\Gamma_{q}(\beta+1)}(t-a)_{q}^{\beta}\right){ }_{q} E_{\alpha+\beta}(c, t-a) \\
& +\left(\left\|B_{0}\right\|+\left\|B_{1}\right\|+2 L_{2}\right)\left(1+\frac{\left\|A_{1}\right\|+L_{1}}{\Gamma_{q}(\alpha+1)}(t-a)_{q}^{\alpha}\right) q_{\alpha+\beta, \beta+1}(c, t-a)<\frac{\varepsilon}{\delta}
\end{aligned}
$$

where $c=\left(\left\|A_{0}\right\|+\left\|A_{1}\right\|+2 L_{1}\right)\left(\left\|B_{0}\right\|+\left\|B_{1}\right\|+2 L_{2}\right)$.

Proof. From Theorem 4 in [19], it is easy to see that $(x, y): \mathbb{T}_{\tau a} \times \mathbb{T}_{\tau a} \longrightarrow \mathbb{R}^{n} \times \mathbb{R}^{n}$ is a solution of system (6) if and only if

$$
\left\{\begin{array}{l}
x(t)=\phi(\alpha)+\frac{1}{\Gamma_{q}(\alpha)} \int_{a}^{t}(t-\mathrm{qs})_{q}^{\alpha-1}\left[A_{0} y(s)+A_{1} y(\tau s)+f(s, y(s), y(\tau s))\right] \nabla_{q} s \\
y(t)=\psi(\alpha)+\frac{1}{\Gamma_{q}(\beta)} \int_{a}^{t}(t-\mathrm{qs})_{q}^{\beta-1}\left[B_{0} x(s)+B_{1} x(\tau s)+g(s, x(s), x(\tau s))\right] \nabla_{q} s \\
x(t)=\phi(t), y(t)=\psi(t), t \in \mathbb{\mathbb { V }}_{\tau} .
\end{array}\right.
$$

For $t \in \mathbb{T}_{a}$, we have, by (55), that

$$
\begin{aligned}
\|x(t)\| \leq & \|\phi(a)\|+\frac{1}{\Gamma_{q}(\alpha)} \int_{a}^{t}(t-\mathrm{qs})_{q}^{\alpha-1}\left\|A_{0} y(s)+A_{1} y(\tau s)+f(s, y(s) y(\tau s))\right\| \nabla_{q} s \\
\leq & \|\phi\|+\frac{\left\|A_{0}\right\|}{\Gamma_{q}(\alpha)} \int_{a}^{t}(t-\mathrm{qs})_{q}^{\alpha-1}\|y(s)\| \nabla_{q} s+\frac{\left\|A_{1}\right\|}{\Gamma_{q}(\alpha)} \int_{a}^{t}(t-\mathrm{q})_{q}^{\alpha-1}\|y(\tau s)\| \nabla_{q} s \\
& +\frac{1}{\Gamma_{q}(\alpha)} \int_{a}^{t}(t-\mathrm{qs})_{q}^{\alpha-1} \| f(s, y(s), y(s)) k r q s \\
\leq & \|\phi\|+\frac{\left\|A_{0}\right\|+L_{1}}{\Gamma_{q}(\alpha)} \int_{a}^{t}(t-\mathrm{qs})_{q}^{\alpha-1}\|y(s)\| \nabla_{q} s+\frac{\left\|A_{1}\right\|+L_{1}}{\Gamma_{q}(\alpha)} \int_{a}^{t}(t-\mathrm{qs})_{q}^{\alpha-1}\|y(\tau s)\| \nabla_{q} s
\end{aligned}
$$




$$
\begin{aligned}
& \leq\|\phi\|+\frac{\left\|A_{0}\right\|+L_{1}}{\Gamma_{q}(\alpha)} \int_{a}^{t}(t-\mathrm{qs})_{q}^{\alpha-1}\|y(s)\| \nabla_{q} s \\
& +\frac{\left\|A_{1}\right\|+L_{1}}{\Gamma_{q}(\alpha)} \int_{a}^{t}(t-\mathrm{qs})_{q}^{\alpha-1}\left[\sup _{\theta \in \unlhd_{\tau}}\|y(\theta s)\|+\|\psi\|\right] \nabla_{q} s \\
& =\|\phi\|+\frac{\|\psi\|\left(\left\|A_{1}\right\|+L_{1}\right)}{\Gamma_{q}(\alpha+1)}(t-a)_{q}^{\alpha}+\frac{\left\|A_{0}\right\|+L_{1}}{\Gamma_{q}(\alpha)} \int_{a}^{t}(t-\mathrm{qs})_{q}^{\alpha-1}\|y(s)\| \nabla_{q} s \\
& +\frac{\left\|A_{1}\right\|+L_{1}}{\Gamma_{q}(\alpha)} \int_{a}^{t}(t-\mathrm{qs})_{q}^{\alpha-1} \sup _{\theta \in \unlhd_{\tau}}\|y(\theta s)\| \nabla_{q} s,
\end{aligned}
$$

where $\mathbb{J}_{\tau}=\left\{\left\{\tau, \tau q^{-1}, \ldots, 1\right\}\right.$. Similarly, we can obtain

$$
\begin{gathered}
\|y(t)\| \leq\|\psi\|+\frac{\|\phi\|\left(\left\|B_{1}\right\|+L_{2}\right)}{\Gamma_{q}(\beta+1)}(t-a)_{q}^{\beta}+\frac{\left\|B_{0}\right\|+L_{2}}{\Gamma_{q}(\beta)} \int_{a}^{t}(t-\mathrm{qs})_{q}^{\beta-1}\|x(s)\| \nabla_{q} s \\
+\frac{\left\|B_{1}\right\|+L_{2}}{\Gamma_{q}(\beta)} \int_{a}^{t}(t-\mathrm{qs})_{q}^{\beta-1} \sup _{\theta \in \unlhd_{\tau}}\|x(\theta s)\| \nabla_{q} s .
\end{gathered}
$$

Let $g_{1}(t)=\|\phi\|+\|\psi\|\left(\left\|A_{1}\right\|+L_{1}\right) / \Gamma_{q}(\alpha+1)(t-a)_{q}^{\alpha}$ and $g_{2}(t)=\|\psi\|+\|\phi\|\left(\left\|B_{1}\right\|+L_{2}\right) / \Gamma_{q}(\beta+1)(t-a)_{q}^{\beta} ;$ then, $g_{1}$ and $g_{2}$ are two nondecreasing functions.

Set $\bar{x}(t)=\sup _{\theta \in \unlhd_{\tau}}\|x(\theta t)\|$ and $\bar{y}(t)=\sup _{\theta \in \unlhd_{\tau}}\|y(\theta t)\|$; then, by (56), we obtain

$$
\begin{aligned}
\bar{x}(t) \leq & g_{1}(t)+\frac{\left\|A_{0}\right\|+L_{1}}{\Gamma_{q}(\alpha)} \int_{a}^{t}(t-\mathrm{qs})_{q}^{\alpha-1} \bar{y}(s) \nabla_{q} s \\
& +\frac{\left\|A_{1}\right\|+L_{1}}{\Gamma_{q}(\alpha)} \int_{a}^{t}(t-\mathrm{qs})_{q}^{\alpha-1} \bar{y}(s) \nabla_{q} s \\
= & g_{1}(t)+\frac{\left\|A_{0}\right\|+\left\|A_{1}\right\|+2 L_{1}}{\Gamma_{q}(\alpha)} \int_{a}^{t}(t-\mathrm{qs})_{q}^{\alpha-1} \bar{y}(s) \nabla_{q} s \\
= & g_{1}(t)+\left(\left\|A_{0}\right\|+\left\|A_{1}\right\|+2 L_{1}\right)_{q} \nabla_{a}^{-\alpha} \bar{y}(t) .
\end{aligned}
$$

\section{Similarly, we obtain}

$$
\bar{y}(t) \leq g_{2}(t)+\left(\left\|B_{0}\right\|+\left\|B_{1}\right\|+2 L_{2}\right)_{q} \nabla_{a}^{-\beta} \bar{x}(t) .
$$

Hence, by (53) and (54) and applying the result of Corollary 1, we have

$$
\begin{aligned}
\|x(t)\| \leq & \bar{x}(t) \leq g_{1}(t)_{q} E_{\alpha+\beta}(c, t-a) \\
& +\left(\left\|A_{0}\right\|+\left\|A_{1}\right\|+2 L_{1}\right) g_{2}(t)_{q} e_{\alpha+\beta, \alpha+1}(c, t-a) \\
= & \left(1+\frac{\left\|A_{1}\right\|+L_{1}}{\Gamma_{q}(\alpha+1)}(t-a)_{q}^{\alpha}\right)\|(\phi, \psi)\|_{q} E_{\alpha+\beta}(c, t-a)
\end{aligned}
$$




$$
\begin{aligned}
& +\left(\left\|A_{0}\right\|+\left\|A_{1}\right\|+2 L_{1}\right)\left(1+\frac{\left\|B_{1}\right\|+L_{2}}{\Gamma_{q}(\beta+1)}(t-a)_{q}^{\beta}\right)\|(\phi, \psi)\|_{q} e_{\alpha+\beta, \alpha+1}(c, t-a) \\
\leq & \delta\left(1+\frac{\left\|A_{1}\right\|+L_{1}}{\Gamma_{q}(\alpha+1)}(t-a)_{q}^{\alpha}\right)_{q} E_{\alpha+\beta}(c, t-a) \\
& +\delta\left(\left\|A_{0}\right\|+\left\|A_{1}\right\|+2 L_{1}\right)\left(1+\frac{\left\|B_{1}\right\|+L_{2}}{\Gamma_{q}(\beta+1)}(t-a)_{q}^{\beta}\right)_{q} e_{\alpha+\beta, \alpha+1}(c, t-a)<\varepsilon,
\end{aligned}
$$

and

$$
\begin{aligned}
\|y(t)\| \leq & \bar{y}(t) \leq g_{2}(t)_{q} E_{\alpha+\beta}(c, t-a) \\
& +\left(\left\|B_{1}\right\|+\left\|B_{0}\right\|+2 L_{2}\right) g_{1}(t)_{q} e_{\alpha+\beta, \beta+1}(c, t-a) \\
= & \left(\|\psi\|+\frac{\|\phi\|\left(\left\|B_{1}\right\|+L_{2}\right)}{\Gamma_{q}(\beta+1)}(t-a)_{q}^{\beta}\right)_{q} E_{\alpha+\beta}(c, t-a) \\
& +\left(\left\|B_{1}\right\|+\left\|B_{0}\right\|+2 L_{2}\right)\left(\|\phi\|+\frac{\|\psi\|\left(\left\|A_{1}\right\|+L_{1}\right)}{\Gamma_{q}(\alpha+1)}(t-a)_{q}^{\alpha}\right)_{q} e_{\alpha+\beta, \beta+1}(c, t-a) . \\
\leq & \delta\left(1+\frac{\left\|B_{1}\right\|+L_{2}}{\Gamma_{q}(\beta+1)}(t-a)_{q}^{\beta}\right)_{q} E_{\alpha+\beta}(c, t-a) \\
& +\delta\left(\left\|B_{1}\right\|+\left\|B_{0}\right\|+2 L_{2}\right)\left(1+\frac{\left\|A_{1}\right\|+L_{1}}{\Gamma_{q}(\alpha+1)}(t-a)_{q}^{\alpha}\right)_{q} e_{\alpha+\beta, \beta+1}(c, t-a)<\varepsilon .
\end{aligned}
$$

Remark 1. In [34], Lyu and Vong proposed and analyzed a scheme on nonuniform mesh for solving the $q$-Volterra equation which provides the same solution to the $q$-fractional initial value problem. We will apply the proposed scheme as in (3.12) in [32] to solve Example 1.

\section{Example}

Example 1. Consider the nonlinear delay $q$-fractional differential difference coupled system:

$$
\begin{cases}{ }_{q} C_{a}^{0.7} x(t)=0.7 y(t)+\sin \left(\frac{1}{8} y(\tau t)\right), & t \in[a, 1]_{q}, \\ { }_{q} C_{a}^{0.5} y(t)=0.8 x(t)+\arctan \left(\frac{1}{9} x(\tau t)\right), & t \in[a, 1]_{q},\end{cases}
$$

where $\alpha=0.7, \beta=0.5, q=0.6, a=q^{9}=0.6^{9}, \tau=q^{9}=0.6^{9}$, $f(t, y(t), y(\tau t))=\sin ((1 / 8) y(\tau t)), \quad g(t, x(t), x(\tau t))=$ $\arctan ((1 / 9) x(\tau t)), \quad \phi(t)=0.07, \quad$ and $\psi(t)=0.09$, and $t \in \mathbb{\mathbb { T }}_{\tau}=\left\{0.6^{18}, 0.6^{17}, \ldots, 0.6^{9}\right\}$. 


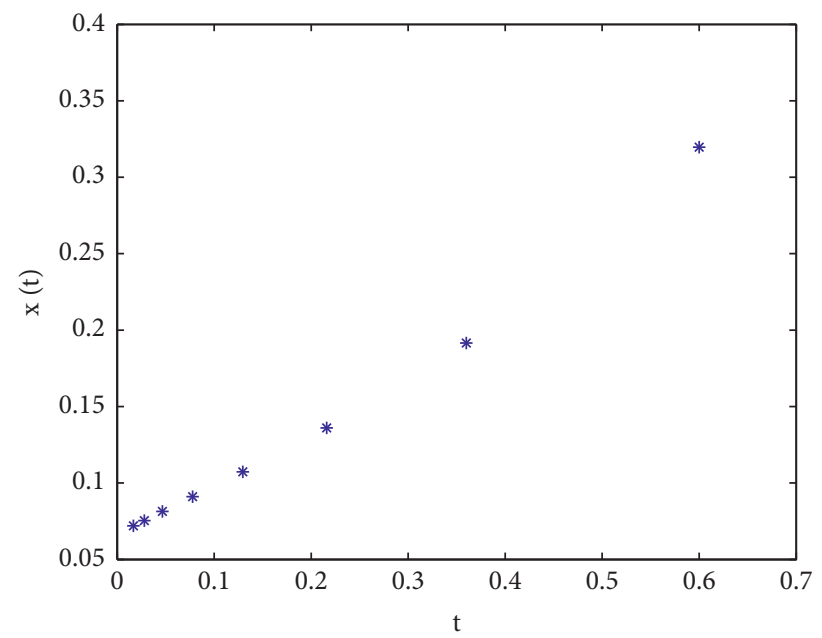

FIgURE 1: Behavior of the solution of system $(62)$ in the $(t, x)$ plane within $T=0.6 \mathrm{~s}$.

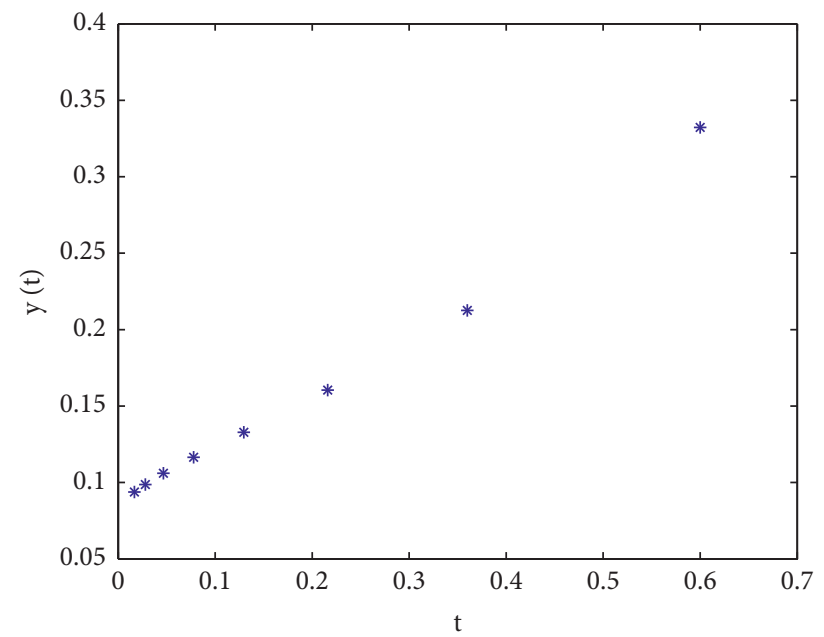

FIgURE 2: Behavior of the solution of system $(62)$ in the $(t, y)$ plane within $T=0.6 \mathrm{~s}$.

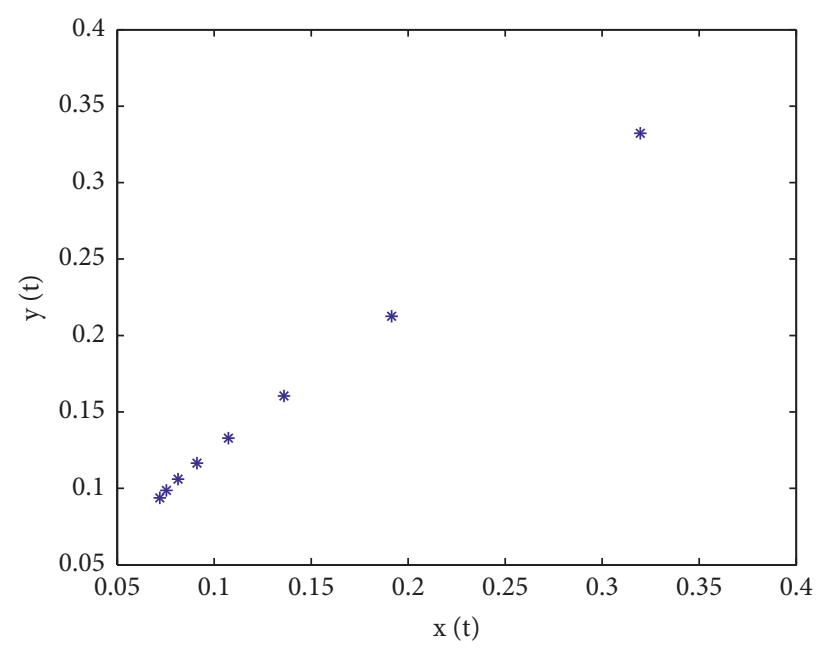

Figure 3: Phase plot of (62). 
Let $\delta=0.1 \quad$ and $\quad \epsilon=0.5$ Obviously, $\|(\phi, \psi)\|=\max \{0.07,0.09\}<0.1=\delta$. We can see that $f, g$ satisfy conditions (H2) and (H1) with $L_{1}=1 / 8$ and $L_{2}=1 / 9$. Moreover, we have $\left\|A_{0}\right\|=0.7,\left\|A_{1}\right\|=0,\left\|B_{0}\right\|=0.8$, and
$\left\|B_{1}\right\|=0$, and (H3) holds. In the following, the aim is to validate the FTS conditions (53) and (54), w.r.t. $\{\delta, \epsilon, T\}$. By using Matlab (the pseudocode to compute different values of $\Gamma_{q}(\alpha)$, see $\left.[35]\right)$, when $t=0.6 \in[a, 1]_{q}$,

$$
\begin{aligned}
& \left(1+\frac{\left\|A_{1}\right\|+L_{1}}{\Gamma_{q}(\alpha+1)}(t-a)_{q}^{\alpha}\right){ }_{q} E_{\alpha+\beta}(c, t-a) \\
& +\left(\left\|A_{0}\right\|+\left\|A_{1}\right\|+2 L_{1}\right)\left(1+\frac{\left\|B_{1}\right\|+L_{2}}{\Gamma_{q}(\beta+1)}(t-a)_{q}^{\beta}\right){ }_{q} e_{\alpha+\beta, \alpha+1}(c, t-a) \\
& \approx 3.4304<5=\frac{\varepsilon}{\delta},
\end{aligned}
$$

and

$$
\begin{aligned}
& \left(1+\frac{\left\|B_{1}\right\|+L_{2}}{\Gamma_{q}(\beta+1)}(t-a)_{q}^{\beta}\right)_{q} E_{\alpha+\beta}(c, t-a) \\
& +\left(\left\|B_{0}\right\|+\left\|B_{1}\right\|+2 L_{2}\right)\left(1+\frac{\left\|A_{1}\right\|+L_{1}}{\Gamma_{q}(\alpha+1)}(t-a)_{q}^{\alpha}\right){ }_{q} e_{\alpha+\beta, \beta+1}(c, t-a) \\
& \approx 4.8461<5=\frac{\varepsilon}{\delta} .
\end{aligned}
$$

Thus, we obtain that the estimated time of FTS is $T=0.6$. Within given parameters, we can observe the finite-time behavior. In Figures 1 and 2, we can see that, within the finite-time $T=0.6 s,\|(\phi, \psi)\|=0.09<\delta=0.1$, the norm $\|(x, y)\|$ of solution $(x(t), y(t))$ does not exceed $\varepsilon=0.5$ which supposes the theorem numerically. The phase plot of (62) is shown in Figure 3.

\section{Conclusion}

The problem of finite-time stability of coupled $q$-fractional difference delay systems is emphasized in this work. For this, we obtained a generalized coupled q-fractional Gronwall inequality, and by applying this inequality, a novel and easy to verify sufficient conditions have been provided in this paper to determine the finite-time stability of the solutions for the considered system. Finally, an example is given to illustrate the effectiveness and feasibility of our criterion.

In the future, we will consider more $q$-fractional difference systems, such as $q$-fractional difference singular systems or $q$-fractional difference uncertain systems, and we will study the problems of finite-time stability, and the Ulam-Hyers stability for these systems.

\section{Data Availability}

No data were used to support this study.

\section{Conflicts of Interest}

The authors declare that they have no conflicts of interest.

\section{Authors' Contributions}

The authors contributed equally to this paper. All authors read and approved the final manuscript.

\section{Acknowledgments}

This work was supported by Natural Science Foundation of China (11571136).

\section{References}

[1] R. Floreanini and L. Vinet, "Quantum symmetries of q-difference equations," Journal of Mathematical Physics, vol. 36, no. 6, pp. 3134-3156, 1995.

[2] M. Marin, "On existence and uniqueness in thermoelasticity of micropolar bodies," Comptes rendus de l'Académie des Sciences, vol. 321, no. 12, pp. 475-480, 1995.

[3] R. Finkelstein and E. Marcus, "Transformation theory of the q-oscillator," Journal of Mathematical Physics, vol. 36, no. 6, pp. 2652-2672, 1995.

[4] M. Marin, "Lagrange identity method for microstretch thermoelastic materials," Journal of Mathematical Analysis and Applications, vol. 363, no. 1, pp. 275-286, 2010.

[5] T. Ernst, A Comprehensive Treatment of Q-Calculus, Birkhäuser, Basel, Switzerland, 2012. 
[6] N. R. O. Bastos, R. A. C. Ferreira, R. A. C. Ferreira, and F. M. Torres, "Necessary optimality conditions for fractional difference problems of the calculus of variations," Discrete \& Continuous Dynamical Systems-A, vol. 29, no. 2, pp. 417-437, 2011.

[7] F. M. Atici and P. W. Eloe, "Initial value problems in discrete fractional calculus," Proceedings of the American Mathematical Society, vol. 137, no. 3, pp. 981-989, 2009.

[8] C. S. Goodrich, "Continuity of solutions to discrete fractional initial value problems," Computers \& Mathematics with Applications, vol. 59, no. 11, pp. 3489-3499, 2010.

[9] G. A. Anastassiou, "Nabla discrete fractional calculus and nabla inequalities," Mathematical and Computer Modelling, vol. 51, no. 5-6, pp. 562-571, 2010.

[10] F. Jarad, T. Abdeljawad, and D. Baleanu, "Stability of $q$ fractional non-autonomous systems," Nonlinear Analysis: Real World Applications, vol. 14, no. 1, pp. 780-784, 2013.

[11] T. Abdeljawad and D. Baleanu, "Caputo q-fractional initial value problems and a q-analogue Mittag-Leffler function," Communications in Nonlinear Science and Numerical Simulation, vol. 16, no. 12, pp. 4682-4688, 2011.

[12] Z. S. I. Mansour, "Linear sequential $q$-difference equations of fractional order," Fractional Calculus and Applied Analysis, vol. 12, no. 2, pp. 159-178, 2009.

[13] X. Li, Z. Han, and X. Li, "Boundary value problems of fractional q-difference Schröinger equations," Applied Mathematics Letters, vol. 46, pp. 100-105, 2015.

[14] J. Mao, Z. Zhao, and C. Wang, "The unique iterative positive solution of fractional boundary value problem with q-difference," Applied Mathematics Letters, vol. 100, p. 106002, 2020.

[15] Y. Liang, H. Yang, and H. Li, "Existence of positive solutions for the fractional $q$-difference boundary value problem," Advances in Difference Equations, vol. 2020, p. 416, 2020.

[16] J. Wang and C. Bai, "Finite-time stability of $q$-fractional damped difference systems with time delay," AIMS Mathematics, vol. 6, no. 11, pp. 12011-12027, 2021.

[17] M. H. Annaby and Z. S. Mansour, " $q$-fractional calculus and equations," Lecture Notes in Mathematics, vol. 2056, 2012.

[18] T. Abdeljawad and J. Alzabut, "The $q$-fractional analogue for Gronwall-type inequality," Journal of Function Spaces, vol. 2013, p. 543839, 2013.

[19] T. Abdeljawad, J. Alzabut, and D. Baleanu, “A generalized $q$-fractional Gronwall inequality and its applications to nonlinear delay $q$-fractional difference systems," Journal of Inequalities and Applications, vol. 2016, p. 240, 2016.

[20] Z. Sun, Z. Liu, and X. Zhang, "New results on global stabilization for time-delay nonlinear systems with low-order and high-order growth conditions," International Journal of Robust and Nonlinear Control, vol. 25, no. 6, pp. 878-899, 2015.

[21] X. Wang and Z. Xiang, "Global finite-time stabilisation for a class of nonlinear systems in the p-normal form via output feedback," International Journal of Systems Science, vol. 51, no. 9, pp. 1604-1621, 2020.

[22] X. Wang, S. Huang, and Z. Xiang, "Output feedback finitetime stabilization of a class of nonlinear time-delay systems in the p-normal form," International Journal of Robust and Nonlinear Control, vol. 30, pp. 1-15, 2020.

[23] A. Modiri and S. Mobayen, "Adaptive terminal sliding mode control scheme for synchronization of fractional-order uncertain chaotic systems," ISA Transactions, vol. 105, pp. 33-50, 2020.

[24] O. Mofid, S. Mobayen, and M. H. Khooban, "Sliding mode disturbance observer control based on adaptive synchronization in a class of fractional-order chaotic systems," International Journal of Adaptive Control and Signal Processing, vol. 33, pp. 1-13, 2018.

[25] H. Karami, S. Mobayen, M. Lashkari, F. Bayat, and A. Chang, "LMI-Observer-based stabilizer for chaotic systems in the existence of a nonlinear function and perturbation," Mathematics, vol. 10, 2021.

[26] S. M. Esmaeilzadeh, M. Golestani, and S. Mobayen, "Chattering-free fault-tolerant attitude control with fast fixed-time convergence for flexible spacecraft," International Journal of Control, Automation and Systems, vol. 19, pp. 1-10, 2021.

[27] M. P. Lazarevic and A. M. Spasic, "Finite-time stability analysis of fractional order time-delay systems: Gronwall approach," Mathematical and Computer Modelling, vol. 49, pp. $475-481,2009$.

[28] V. N. Phat and N. T. Thanh, "New criteria for finite-time stability of nonlinear fractional-order delay systems: a Gronwall inequality approach," Applied Mathematics Letters, vol. 83, pp. 169-175, 2018.

[29] R. Wu, Y. Lu, and L. Chen, "Finite-time stability of fractional delayed neural networks," Neurocomputing, vol. 149, pp. 700-707, 2015.

[30] M. Li and J. Wang, "Exploring delayed Mittag-Leffler type matrix functions to study finite time stability of fractional delay differential equations," Applied Mathematics and Computation, vol. 324, pp. 254-265, 2018.

[31] F. Du and J.-G. Lu, "Finite-time stability of neutral fractional order time delay systems with Lipschitz nonlinearities," Applied Mathematics and Computation, vol. 375, p. 125079, 2020.

[32] M. Mansour, "An asymptotic expansion of the q-gamma function q(x)," Journal of Nonlinear Mathematical Physics, vol. 13 , no. 4 , pp. 479-483, 2006.

[33] R. A. Adams and C. Essex, Calculus A Complete Course, Pearson Canada, Toronto, Canada, Seventh edition, 2010.

[34] P. Lyn and S. Vong, "An efficient numerical method for q-fractional differential equations," Applied Mathematics Letters, vol. 103, p. 106156, 2020.

[35] N. D. Phuong, F. M. Sakar, S. Etemad, and S. Rezapour, "A novel fractional structure of a multi-order quantum multiintegro-differential problem," Advances in Difference Equations, vol. 2020, no. 1, p. 633, 2020. 